\title{
Dirac's Classical-Quantum Analogy for the Harmonic Oscillator: Classical Aspects in Thermal Radiation Including Zero-Point Radiation
}

\author{
Timothy H. Boyer \\ Department of physics, City College of the City \\ University of New York, New York, New York 10031
}

\begin{abstract}
Dirac's Poisson-bracket-to-commutator analogy for the transition from classical to quantum mechanics assures that for many systems, the classical and quantum systems share the same algebraic structure. The quantum side of the analogy (involving operators on Hilbert space with commutators scaled by Planck's constant $\hbar$ ) not only gives the algebraic structure but also dictates the average values of physical quantities in the quantum ground state. On the other hand, the Poisson brackets of nonrelativistic mechanics, which give only the classical canonical transformations, do not give any values for physical quantities. Rather, one must go outside nonrelativistic classical mechanics in order to obtain a fundamental phase space distribution for classical physics. We assume that the values of physical quantities in classical theory at any temperature depend on the phase space probability distribution which arises from thermal radiation equilibrium including classical zero-point radiation with the scale set by Planck's constant $\hbar$. All mechanical systems in thermal radiation will inherit the constant $\hbar$ from thermal radiation. Here we note the connections between classical and quantum theories (agreement and contrasts) at all temperatures for the harmonic oscillator in one and three spatial dimensions.
\end{abstract}




\section{CLASSICAL-QUANTUM CONNECTIONS FOR THE HARMONIC OSCILLA- TOR}

The formalism of quantum mechanics is very different from that of classical physics. However, in some cases, there are striking parallels between the theories. In 1925, Dirac[1] suggested that the transition from classical to quantum theory could be carried out by replacing the Poisson brackets of classical mechanics by commutation relations for operators with a scale given by Planck's constant $\hbar$. This classical-quantum analogy,[2] taken together with operator symmetrization in the Hamiltonian, assures that the algebraic structure involving the Hamiltonian operator in quantum theory matches the algebraic structure associated with the Hamiltonian function in classical mechanics for many systems. [3] For familiar mechanical systems, such a replacement indeed produces the current quantum theory, and the Dirac classical-quantum analogy is sometimes mentioned in the textbooks. [2] However, the connections between the equilibrium average values of physical quantities in classical and quantum physics involve aspects different from Dirac's analogy. Quantum operators act on Hilbert space in such a way as to determine the average values of physical quantities in the ground state. In classical mechanics, the equilibrium average values are determined by integrations over phase space; however, nonrelativistic classical physics contains no fundamental scale on phase space. Thus any attempt to compare quantum average values with those of some related classical mechanical system requires an extension beyond nonrelativistic classical mechanics.

The classical extension chosen in this article involves the equilibrium of the classical mechanical system in classical electromagnetic thermal radiation. Planck's constant $\hbar$ has a natural place within classical electromagnetic theory as the scale of Lorentz-invariant random classical electromagnetic radiation, classical zero-point radiation. 4] The constant $\hbar$ is then reflected in the spectrum of equilibrium classical thermal radiation giving the Planck spectrum with classical electromagnetic zero-point radiation. [5] Finally, the constant $\hbar$ is reflected in the equilibrium phase space for any mechanical system in equilibrium with thermal radiation. The mechanical system together with the thermal-equilibrium phase space gives a stochastic classical mechanical theory. In this article, we survey the connections (both similarities and differences) for harmonic oscillator systems in one and in three spatial dimensions when described by quantum mechanics or by classical stochastic mechanics. The 
analysis give us a deeper appreciation of both classical and quantum theories.

\section{ALGEBRAIC STRUCTURE: COMMUTATORS VS POISSON BRACKETS}

\section{A. Fundamental Commutator and Poisson Brackets}

Quantum commutators appear in a student's first course in quantum mechanics. [6] The easiest form of quantum mechanics seems to involve the Schroedinger wave function $\psi(x, t)$ for a system of one degree of freedom. In this form, the fundamental quantum commutator for the position operator $\hat{x}$ and momentum operator $\hat{p}_{x}$,

$$
\left[\hat{x}, \hat{p}_{x}\right]=\hat{x} \hat{p}_{x}-\hat{p}_{x} \hat{x}=i \hbar
$$

can be regarded as the operators $x$ and $(\hbar / i) \partial / \partial x$ acting on the function space formed by the wave functions $\psi(x, t)$

$$
\left[\hat{x}, \hat{p}_{x}\right] \psi(x, t)=x \frac{\hbar}{i} \frac{\partial}{\partial x} \psi(x, t)-\frac{\hbar}{i} \frac{\partial}{\partial x}[x \psi(x, t)]=i \hbar \psi(x, t)
$$

On the other hand, classical Poisson brackets, which can be used as the basis for the Hamilto-

nian formulation of classical mechanics, appear in a graduate course in classical mechanics. [7] The classical mechanical description of a system of one degree of freedom involves canonical variables $x$ and $p_{x}$ satisfying the fundamental Poisson bracket relation

$$
\left\{x, p_{x}\right\}=\frac{\partial x}{\partial x} \frac{\partial p_{x}}{\partial p_{x}}-\frac{\partial x}{\partial p_{x}} \frac{\partial p_{x}}{\partial x}=1
$$

where for any two functions $f\left(x, p_{x}\right)$ and $g\left(x, p_{x}\right)$ the Poisson bracket is

$$
\{f, g\}=\frac{\partial f}{\partial x} \frac{\partial g}{\partial p_{x}}-\frac{\partial f}{\partial p_{x}} \frac{\partial g}{\partial x} .
$$

The classical mechanical system is described by a Hamiltonian function involving the canonical coordinate $x$ and canonical momentum $p_{x}$, and the Poisson brackets lead to the generation of infinitesimal canonical transformations. 


\section{B. One-Dimensional Harmonic Oscillator}

\section{Classical Oscillator}

The Hamiltonian describing the one-dimensional harmonic oscillator in classical physics is

$$
H=\frac{p_{x}^{2}}{2 m}+\frac{1}{2} m \omega_{0}^{2} x^{2}
$$

The Hamiltonian is the generator of canonical time translations, and hence gives Hamilton's equations of motion in the classical case,

$$
\frac{d x}{d t}=\{x, H\}=\frac{\partial x}{\partial x} \frac{\partial H}{\partial p_{x}}-\frac{\partial x}{\partial p_{x}} \frac{\partial H}{\partial x}=\frac{p_{x}}{m}
$$

and

$$
\frac{d p_{x}}{d t}=\left\{p_{x}, H\right\}=\frac{\partial p_{x}}{\partial x} \frac{\partial H}{\partial p_{x}}-\frac{\partial p_{x}}{\partial p_{x}} \frac{\partial H}{\partial x}=-m \omega_{0}^{2} x .
$$

The solutions of the equations of motion are

$$
\begin{gathered}
x(t)=x(0) \cos \omega_{0} t+\left(\frac{p_{x}(0)}{m \omega_{0}}\right) \sin \omega_{0} t, \\
p_{x}(t)=-m \omega_{0} x(0) \sin \omega_{0} t+p_{x}(0) \cos \omega_{0} t .
\end{gathered}
$$

\section{Quantum Oscillator}

The quantum mechanical description of the harmonic oscillator in the Heisenberg picture follows the same pattern as given above for the classical oscillator. The Hamiltonian now involves operators $\hat{x}$ and $\hat{p}$,

$$
\hat{H}=\frac{\hat{p}_{x}^{2}}{2 m}+\frac{1}{2} m \omega_{0}^{2} \hat{x}^{2}
$$

The equations of motion for the operators $\hat{x}$ and $\hat{p}_{x}$ in the Heisenberg picture are [8]

$$
i \hbar \frac{d \hat{x}}{d t}=[\hat{x}, \hat{H}]=\hat{x} \hat{H}-\hat{H} \hat{x}=i \hbar \frac{p_{x}}{m}
$$

and

$$
i \hbar \frac{d \hat{p}_{x}}{d t}=\left[\hat{p}_{x}, \hat{H}\right]=\hat{p}_{x} \hat{H}-\hat{H} \hat{p}_{x}=-i \hbar m \omega_{0}^{2} \hat{x}
$$


The solutions of the equations of motion for the Heisenberg operators are exactly parallel to the classical case,

$$
\begin{aligned}
\hat{x}(t) & =\hat{x}(0) \cos \omega_{0} t+\left(\frac{\hat{p}_{x}(0)}{m \omega_{0}}\right) \sin \omega_{0} t, \\
\hat{p}_{x}(t) & =-m \omega_{0} \hat{x}(0) \sin \omega_{0} t+\hat{p}_{x}(0) \cos \omega_{0} t .
\end{aligned}
$$

\section{Three-Dimensional Oscillator and Angular Momentum}

In the case of the three-dimensional oscillator, one can discuss the algebraic structure involving angular momentum in both classical and quantum theories. The Hamiltonian for the three-dimensional isotropic harmonic oscillator takes the form of three independent harmonic oscillators along the $x, y$, and $z$ axes,

$$
H=\sum_{i=1}^{3}\left(\frac{p_{i}^{2}}{2 m}+\frac{1}{2} m \omega_{0}^{2} x_{i}^{2}\right),
$$

with the classical Hamiltonian involving canonically conjugate variables $x_{i}$ and $p_{i}$, and the analogous quantum Hamiltonian $\hat{H}$ involving quantum operators $\hat{x}_{i}$ and $\hat{p}_{i}$.

The $z$-component of angular momentum of the particle in a three-dimensional harmonic potential involves the function $L_{z}=x p_{y}-y p_{x}$ in classical physics, and $\hat{L}_{z}=\hat{x} \hat{p}_{y}-\hat{y} \hat{p}_{x}$ in the quantum formulation. The components of angular momentum around the $x$ - and $y$-axis are formed analogously. Using Poisson brackets, the angular momentum functions

are generators of the 3-dimensional rotation group. The Poisson brackets now involve $x, p_{x}, y, p_{y}, z, p_{z}$. Thus we have

$$
\begin{aligned}
\left\{L_{x}, L_{y}\right\} & =\frac{\partial L_{x}}{\partial x} \frac{\partial L_{y}}{\partial p_{x}}-\frac{\partial L_{x}}{\partial p_{x}} \frac{\partial L_{y}}{\partial x}+\frac{\partial L_{x}}{\partial y} \frac{\partial L_{y}}{\partial p_{y}}-\frac{\partial L_{x}}{\partial p_{y}} \frac{\partial L_{y}}{\partial y}+\frac{\partial L_{x}}{\partial z} \frac{\partial L_{y}}{\partial p_{z}}-\frac{\partial L_{x}}{\partial p_{z}} \frac{\partial L_{y}}{\partial z} \\
& =\frac{\partial\left(y p_{z}-z p_{y}\right)}{\partial z} \frac{\partial\left(z p_{x}-x p_{z}\right)}{\partial p_{z}}-\frac{\partial\left(y p_{z}-z p_{y}\right)}{\partial p_{z}} \frac{\partial\left(z p_{x}-x p_{z}\right)}{\partial z}=x p_{y}-y p_{x}=L_{z}
\end{aligned}
$$

In general, we have in classical mechanics

$$
\left\{L_{i}, L_{j}\right\}=\epsilon_{i j k} L_{k}
$$


On the other hand, within quantum physics, the commutator gives

$$
\begin{aligned}
{\left[\hat{L}_{x}, \hat{L}_{y}\right] } & =\hat{L}_{x} \hat{L}_{y}-\hat{L}_{y} L_{x}=\left(\hat{y} \hat{p}_{z}-\hat{z} \hat{p}_{y}\right)\left(\hat{z} \hat{p}_{x}-\hat{x} \hat{p}_{z}\right)-\left(\hat{z} \hat{p}_{x}-\hat{x} \hat{p}_{z}\right)\left(\hat{y} \hat{p}_{z}-z \hat{p}_{y}\right) \\
& =\hat{y} \hat{p}_{z} \hat{z} \hat{p}_{x}+\hat{z} \hat{p}_{y} \hat{x} \hat{p}_{z}-\hat{z} \hat{p}_{x} \hat{y} \hat{p}_{z}-\hat{x} \hat{p}_{z} \hat{z} \hat{p}_{Y} \\
& =\hat{y}\left[\hat{p}_{z}, \hat{z}\right] \hat{p}_{x}-\hat{x}\left[\hat{p}_{z}, \hat{z}\right] \hat{p}_{y}=i \hbar\left(\hat{x} \hat{p}_{y}-\hat{y} \hat{p}_{x}\right)=i \hbar \hat{L}_{z} .
\end{aligned}
$$

In general, we have in quantum mechanics

$$
\left[\hat{L}_{i}, \hat{L}_{j}\right]=\epsilon_{i j k} i \hbar \hat{L}_{k}
$$

Thus in both the classical and quantum theories the angular momentum is associated with the algebra of the infinitesimal generators of the rotation group.

In addition, it is easy to prove that the square of the angular momentum $L^{2}=L_{x}^{2}+L_{y}^{2}+L_{z}^{2}$ satisfies

$$
\left\{L^{2}, L_{i}\right\}=0
$$

for the classical Poisson brackets, and that the quantum operator $\hat{L}^{2}=\hat{L}_{x}^{2}+\hat{L}_{y}^{2}+\hat{L}_{z}^{2}$ commutes with all the angular momentum operators

$$
\left[\hat{L}^{2}, \hat{L}_{i}\right]=0
$$

Thus the square of the angular momentum acts as a Casimir operator in the Lie algebra of the generators of the three-dimensional rotation group. [9] The values taken by the Casimir operator $L^{2}$ correspond to $l(l+1)$ in the classical theory, and $\hbar^{2} l(l+1)$ in the quantum theory.

\section{NUMERICAL VALUES: HILBERT SPACE VS PHASE SPACE}

\section{A. Average Values in Classical and Quantum Mechanics}

Dirac emphasized that the association of the Poisson bracket with the commutation would give parallel algebraic structures in classical and quantum theories. [1] However, the theories involve more than simply their algebraic structure. In order to obtain equilibrium numerical values for physical quantities, the classical theory must evaluate the canonical variables over the appropriate phase space probability distribution. For motion in one spatial dimension, 
the phase space distribution $P\left(x, p_{x}\right)$ gives the average value of the quantity $f\left(x, p_{x}\right)$ as

$$
\left\langle f\left(x, p_{x}\right)\right\rangle_{\text {classical }}=\int_{-\infty}^{\infty} d x \int_{-\infty}^{\infty} d p_{x} f\left(x, p_{x}\right) P\left(x, p_{x}\right) .
$$

Such an evaluation is familiar from classical statistical mechanics where the phase space distribution corresponds to that of thermal equilibrium. If the classical phase space involves simply fixed initial conditions, the probability distribution reduces to a single point on the phase space.

On the other hand, the quantum theory at zero temperature obtains average values by applying the quantum operators to Hilbert space as the expectation value

$$
\left\langle f\left(\hat{x}, \hat{p}_{x}\right)\right\rangle_{\text {quantum }}=\int_{-\infty}^{\infty} d x \psi^{*}(x, t) f\left(x, \frac{\hbar}{i} \frac{\partial}{\partial x}\right) \psi(x, t) .
$$

We notice that quantum theory contains the constant $\hbar$ attached to the fundamental commutator in Eq. (2), and this constant will serve as a scale for all the operator expectation values evaluated on the Hilbert space. For example, if we introduce the raising and lowering operators $\hat{a}$ and $\hat{a}^{+}$where[10]

$$
\hat{a}=\sqrt{\frac{m \omega_{0}}{2 \hbar}}\left(\hat{x}+\frac{i \hat{p}_{x}}{m \omega_{0}}\right) \text { and } \hat{a}^{+}=\sqrt{\frac{m \omega_{0}}{2 \hbar}}\left(\hat{x}-\frac{i \hat{p}_{x}}{m \omega_{0}}\right),
$$

then the quantum Hamiltonian in Eq. (9) can be rewritten as

$$
H=\hbar \omega_{0}\left(\hat{a}^{+} \hat{a}+\frac{1}{2}\right)
$$

One can show[11] that the operator $\hat{a}^{+}$acting on the vacuum state can generate states in Hilbert space with energies $(n+1 / 2) \hbar \omega_{0}$ where $n=0,1,2, \ldots$

The average energy of the classical oscillator is obtained by evaluating the values of position and momentum over the phase space which describes the classical system. However, nonrelativistic classical mechanics has nothing to say about a fundamental phase space distribution $P\left(x, p_{x}\right)$.

\section{B. Traditional Contrast Between Classical and Quantum Theories}

It is exactly at this point that the traditional treatments of classical theory and quantum theory part company. Planck's constant $\hbar$ does not appear in the classical mechanical algebra of infinitesimal canonical transformations, and there is no fundamental role for the 
constant in traditional nonrelativistic classical mechanical phase space distributions. Thus the traditional claim arises that Planck's constant $\hbar$ appears in quantum theory, but not in classical theory. Indeed, Goldstein's classical mechanics text book points out the $\mathrm{SO}(4)$ algebraic symmetry of a classical particle in a Coulomb or Kepler potential, [12] but the classical mechanics text book goes no further, while Pauli[13] exploited this same symmetry in quantum theory in order to derive the Balmer spectrum for hydrogen in 1926.

There is a striking contrast between the classical and quantum systems. The quantum system contains a scale parameter $\hbar$ attached to the operators of the theory which arises from the fundamental operator commutator. This scale will produce the ground state energy value for the quantum harmonic oscillator on Hilbert space. The classical mechanical situation is completely different. The classical mechanical system contains no scale within the canonical transformation formalism produced by the Poisson brackets. The values of quantities in the classical mechanical system follow from the assumed phase space distribution.

\section{PHASE SPACE IN CLASSICAL THEORY WITH CLASSICAL ELECTRO- MAGNETIC ZERO-POINT RADIATION}

\section{A. A Closer Classical Approximation to Quantum Physics}

We are interested in the connections between classical and quantum theories when the classical theory is not simply nonrelativistic classical mechanics with no scale $\hbar$, but rather is the classical theory which comes as close as possible to approximating quantum theory. We must go outside nonrelativistic classical mechanic in order to accomplish this aim. One possible extension turns to classical electromagnetic theory. [14]

The arguments leading to an extension into classical electromagnetic theory are as follows. Small bits of matter in a fluid at room temperature are observed to perform a random Brownian motion, presumably forced into motion by collisions with the invisible molecules of the solution. It is therefore assumed that, in equilibrium, a small harmonic oscillator would be forced into thermal motion by colliding gas molecules. Even if all the gas molecules were removed, a charged harmonic oscillator would still come to thermal equilibrium with the ambient thermal radiation. Indeed, Planck's calculation[15] at the end of the 19th century showed that a classical charged harmonic oscillator acquires an average energy equal 
to the average energy per normal mode of the surround random classical radiation at the frequency $\omega_{0}$ of the oscillator. In the limit as the temperature is decreased to zero, the charged harmonic oscillator would be in equilibrium with the random radiation which exists at zero temperature. This random radiation at the zero of temperature is termed classical electromagnetic zero-point radiation. The Casimir force[16] between two uncharged parallel conducting plates depends upon all the radiation surrounding the plates, and experimental measurements[17] show that at zero temperature, the Casimir force does not vanish. Rather, the Casimir force can be explained quantitatively by the existence of random classical radiation with an energy spectrum $\mathcal{E}_{\omega}=(1 / 2) \hbar \omega$ per normal mode.[18] Therefore within a purely classical theory, one must assume that all matter exists in ambient classical electromagnetic thermal radiation which enforces an equilibrium phase space distribution on any mechanical system. At zero temperature, classical thermal radiation is classical zero-point radiation with a Lorentz-invariant spectrum and a scale set by Planck's constant $\hbar$. The existence of classical zero-point radiation at zero temperature is sufficient to allow a classical derivation of the full Planck radiation spectrum (including zero-point radiation) at positive temperature. [5]

The behavior of a classical mechanical system, such as a harmonic oscillator, which interacts with classical thermal radiation will come to equilibrium with a phase space distribution which reflects the randomness of the thermal radiation. Even if one takes the limit as the interaction between the oscillator and the thermal radiation becomes ever smaller, the phase space distribution for the mechanical system will remain unchanged, though the time required to reach equilibrium will increase as the interaction strength decreases. Indeed this situation is familiar for systems in thermal equilibrium where the coupling between thermodynamic systems can be made arbitrarily small and yet the equilibrium phase space distribution for fixed temperature $T$ remains the same, independent of the (small) size of the inter-system coupling. Thus in equilibrium with classical thermal radiation, any classical mechanical system will acquire a phase space distribution which reflects the random character of the thermal radiation and contains the scale factor $\hbar$ arising from the scale of classical electromagnetic zero-point radiation. 


\section{B. Classical Phase Space from Classical Thermal Radiation Including Zero-Point}

\section{Radiation}

In equilibrium, the equation of motion for the harmonic oscillator must include driving by classical thermal radiation with the Planck spectrum including zero-point radiation, corresponding to an energy per normal mode $\mathcal{E}(\omega, T)=\left(\hbar \omega_{0} / 2\right) \operatorname{coth}\left[\hbar \omega_{0} /\left(k_{B} T\right)\right]=$ $\hbar \omega\left[\exp \left(\hbar \omega / k_{B} T\right)-1\right]^{-1}+\hbar \omega / 2$. Thus the correct classical equation of motion obtained by combining equations (6) and (77) is not the purely mechanical expression $m \ddot{x}=-m \omega_{0}^{2} x$ but rather must be written as a Langevin equation involving fluctuation and damping. The oscillating particle is assumed to carry a (small) charge $e$ so that it interacts with random thermal radiation as [19]

$$
m \ddot{x}=-m \omega_{0}^{2} x+m \tau \dddot{x}+e E_{x}^{T}(x, t),
$$

where $\tau=\left(2 e^{2}\right) /\left(3 m c^{3}\right)$ is associated with radiation damping, and $e E_{x}^{T}(x, t)$ is the force on the oscillator due to the random classical thermal radiation. The equation of motion (24) has been solved (for the steady-state solution) many times[20] going back to Planck's

work. 15] The result for the equilibrium phase space distribution $P_{T}\left(x, p_{x}\right)$ for the oscillator in random classical radiation (corresponding to the Planck spectrum at temperature $T$ and including zero-point radiation) is independent of the (small) charge $e$ and takes the form

$$
P_{T}\left(x, p_{x}\right) d x d p_{x}=\frac{1}{2 \pi \hbar \operatorname{coth}\left[\hbar \omega_{0} /\left(k_{B} T\right)\right]} \exp \left(-\frac{p_{x}^{2} / 2 m+m \omega_{0}^{2} x^{2} / 2}{\left(\hbar \omega_{0} / 2\right) \operatorname{coth}\left[\hbar \omega_{0} /\left(k_{B} T\right)\right]}\right) d x d p_{x}
$$

for $-\infty<x<\infty,-\infty<p_{x}<\infty$. At zero temperature $T \rightarrow 0$, the phase space distribution becomes

$$
P_{0}\left(x, p_{x}\right) d x d p_{x}=\frac{1}{2 \pi \hbar} \exp \left(-\frac{p_{x}^{2} / 2 m+m \omega_{0}^{2} x^{2} / 2}{\left(\hbar \omega_{0} / 2\right)}\right) d x d p_{x} .
$$

Thus at zero temperature, the scale $\hbar$ of the classical zero-point radiation which appears in the energy $\hbar \omega / 2$ per radiation normal mode reappears as the scale factor for the oscillator on phase space. The phase space distribution in Eq. (26) is a probability distribution on phase space for the position and momentum of the particle under the influence of classical zero-point radiation. Thus in classical physics with classical electromagnetic zero-point radiation, the canonical transformations determined by the Poisson brackets are separate from the scale of the phase space distribution determined by the oscillator's interaction with classical thermal radiation. 


\section{COMPARISON OF GROUND STATES FOR THE CLASSICAL AND QUAN- TUM OSCILLATORS}

\section{A. One-Dimensional Oscillator}

Since we now have an example of a classical phase space, we can compare and contrast the ground state descriptions for the harmonic oscillator given by the classical and quantum theories. The quantum description is by far the more familiar. The quantum ground state wave function is given by[11]

$$
\psi_{0}(x)=\left(\frac{m \omega_{0}}{\pi \hbar}\right)^{1 / 4} \exp \left(-\frac{m \omega_{0} x^{2}}{2 \hbar}\right) .
$$

It is worth noting that the square of the quantum wave function in Eq. (27) agrees exactly with the classical phase space distribution for $x$ independent of $p_{x}$ found by integrating over the classical phase space distribution in Eq. (26) with respect to $p_{x}$ so as to remove the reference to the momentum $p_{x}$,

$$
\left|\psi_{0}(x)\right|^{2}=\left(\frac{m \omega_{0}}{\pi \hbar}\right)^{1 / 2} \exp \left(-\frac{m \omega_{0} x^{2}}{\hbar}\right)=\int_{-\infty}^{\infty} d p_{x} P_{0}\left(x, p_{x}\right) .
$$

The average value for the $n$th power of the position of the particle is given by

$$
\left\langle\hat{x}^{n}\right\rangle_{0 \text { quantum }}=\int_{-\infty}^{\infty} d x \psi_{0}^{*} x^{n} \psi_{0}=\frac{(2 n) !}{(n !)^{2} 2^{n}}\left(\frac{\hbar}{2 m \omega_{0}}\right)
$$

which is the same as the average value for the classical oscillator over the classical phase space distribution in Eq. (26) arising from the zero-point radiation

$$
\left\langle x^{n}\right\rangle_{0 \text { classical }}=\int_{-\infty}^{\infty} d p_{x} \int_{-\infty}^{\infty} d x x^{n} P_{0}\left(x, p_{x}\right)=\frac{(2 n) !}{(n !)^{2} 2^{n}}\left(\frac{\hbar}{2 m \omega_{0}}\right) .
$$

Similarly, the average values for the powers of momenta agree. Thus the average value for $\hat{p}_{x}^{n}$ is

$$
\left\langle\hat{p}_{x}^{n}\right\rangle_{0 \text { quantum }}=\int_{-\infty}^{\infty} d x \psi_{0}^{*}\left(\frac{\hbar}{i} \frac{d}{d x}\right)^{n} \psi_{0}=\frac{(2 n) !}{(n !)^{2} 2^{n}}\left(\frac{\hbar m \omega_{0}}{2}\right),
$$

which is the same as the average value for the classical oscillator over the phase space distribution arising from the zero-point radiation,

$$
\left\langle p_{x}^{n}\right\rangle_{0 \text { classical }}=\int_{-\infty}^{\infty} d p_{x} \int_{-\infty}^{\infty} d x p_{x}^{n} P_{0}\left(x, p_{x}\right)=\frac{(2 n) !}{(n !)^{2} 2^{n}}\left(\frac{\hbar m \omega_{0}}{2}\right) .
$$


Because both theories agree on the average values $\left\langle\hat{x}^{2}\right\rangle=\left\langle x^{2}\right\rangle$ and $\left\langle\hat{p}_{x}^{2}\right\rangle=\left\langle p_{x}^{2}\right\rangle$, both theories agree on the average value of energy,

$$
\langle\hat{H}\rangle_{0 \text { quantum }}=\int_{-\infty}^{\infty} d x \psi_{0}^{*} H \psi_{0}=\frac{\left\langle\hat{p}_{x}^{2}\right\rangle}{2 m}+\frac{m \omega_{0}^{2}\left\langle\hat{x}^{2}\right\rangle}{2}=\frac{1}{2} \hbar \omega_{0},
$$

and

$$
\langle H\rangle_{0 \text { classical }}=\int_{-\infty}^{\infty} d p_{x} \int_{-\infty}^{\infty} d x H f\left(x, p_{x}\right)=\frac{\left\langle p_{x}^{2}\right\rangle}{2 m}+\frac{m \omega_{0}^{2}\left\langle x^{2}\right\rangle}{2}=\frac{1}{2} \hbar \omega_{0} .
$$

However, the theories differ regarding the fluctuations of energy. For example, the quantum theory involves an energy eigenstate and involves no fluctuations in energy, so that we find

$$
\begin{aligned}
\left\langle\hat{H}^{2}\right\rangle_{0 \text { quantum }} & =\int_{-\infty}^{\infty} d x \psi_{0}^{*} H^{2} \psi_{0}=\left\langle\left(\frac{\hat{p}_{x}^{2}}{2 m}+\frac{1}{2} m \omega_{0}^{2} \hat{x}^{2}\right)^{2}\right\rangle \\
& =\frac{\left\langle\hat{p}_{x}^{4}\right\rangle}{(2 m)^{2}}+\frac{\omega_{0}^{2}}{4}\left(\left\langle\hat{p}_{x}^{2} \hat{x}^{2}\right\rangle+\left\langle\hat{x}^{2} \hat{p}_{x}^{2}\right\rangle\right)+\left(\frac{m \omega_{0}^{2}}{2}\right)^{2}\left\langle\hat{x}^{4}\right\rangle \\
& =\left(\frac{1}{2} \hbar \omega_{0}\right)^{2}=\langle\hat{H}\rangle_{0 \text { quantum }}^{2}
\end{aligned}
$$

since the quantum operators give $\left\langle\hat{x}^{2} \hat{p}_{x}^{2}\right\rangle=\left\langle\hat{p}_{x}^{2} \hat{x}^{2}\right\rangle=-\left\langle\hat{x}^{2}\right\rangle\left\langle\hat{p}_{x}^{2}\right\rangle$. On the other hand, the energy of the classical oscillator indeed fluctuates giving

$$
\begin{aligned}
\left\langle H^{2}\right\rangle_{0 \text { classical }} & =\int_{-\infty}^{\infty} d p_{x} \int_{-\infty}^{\infty} d x H^{2} P_{0}\left(x, p_{x}\right) \\
& =\frac{\left\langle p_{x}^{4}\right\rangle}{(2 m)^{2}}+\frac{\omega_{0}^{2}}{2}\left\langle p_{x}^{2} x^{2}\right\rangle+\left(\frac{m \omega_{0}^{2}}{2}\right)^{2}\left\langle x^{4}\right\rangle=2\langle H\rangle_{0}^{2} \text { classical }
\end{aligned}
$$

since for the classical quantities $\left\langle x^{2} p_{x}^{2}\right\rangle=\left\langle x^{2}\right\rangle\left\langle p_{x}^{2}\right\rangle$. The classical and quantum expressions for $\left\langle\hat{H}^{2}\right\rangle_{0 \text { quantum }}$ and $\left\langle H^{2}\right\rangle_{0 \text { classical }}$ do not agree. In the quantum mechanical ground state, the position and momentum of the particle fluctuate, but the fluctuations are correlated in a special non-classical way[21] such that the energy takes a discrete value; the energy of the quantum oscillator is an eigenvalue for the ground state energy. In contrast, the probabilities of the classical system are fully described by the probability distribution on phase space. For the classical oscillator at zero temperature, the randomness is derived from the randomness of classical zero-point radiation. The energy of the system in equilibrium is a slowly varying quantity which explores the phase space as the oscillator exchanges energy with the classical zero-point radiation, and the energy probability distribution can be obtained from the phase space distribution. [22] The disagreement between the fluctuations of the theories has been 
known for some time. Whether the distinction is experimentally measurable is another matter. So far as I know, no one has viewed the disagreement as appropriate for a possible experimental measurement.

\section{B. Three-Dimensional Harmonic Oscillator}

The agreement and the contrasts between the quantum theory and the classical theory with zero-point radiation can be illustrated further by considering the three-dimensional harmonic oscillator. For this situation, we find interesting contrasts in the treatment of angular momentum.

The Hamiltonian for the three-dimensional isotropic harmonic oscillator takes the form of three independent harmonic oscillators along the $x, y$, and $z$ axes,

$$
H=\sum_{i=1}^{3}\left(\frac{p_{i}^{2}}{2 m}+\frac{1}{2} m \omega_{0}^{2} x_{i}^{2}\right)
$$

with the classical Hamiltonian involving canonically conjugate variables $x_{i}$ and $p_{i}$, and the quantum Hamiltonian $\hat{H}$ involving quantum operators $\hat{x}_{i}$ and $\hat{p}_{i}$. In the ground state at zero temperature, the classical distribution on phase space involves a product probability distribution

$$
P_{0}\left(x, p_{x}, y, p_{y} z, p_{z}\right)=\prod_{i=1}^{3} \frac{1}{(\pi \hbar)^{3}} \exp \left(-\frac{p_{i}^{2} /(2 m)+(1 / 2) m \omega_{0}^{2} x_{i}^{2}}{\hbar \omega_{0} / 2}\right),
$$

and the quantum wave function is given as a product state in Hilbert space

$$
\psi(x, y, z)=\prod_{i=1}^{3}\left(\frac{m \omega_{0}}{\pi \hbar}\right)^{3 / 4} \exp \left(-\frac{m \omega_{0} x_{i}^{2}}{2 \hbar}\right) .
$$

Once again, in the ground state, the quantum wave function squared corresponds to the classical phase space distribution integrated over the momentum variables

$$
|\psi(x, y, z)|^{2}=\int d p_{x} d p_{y} d p_{z} P_{0}\left(x, p_{x}, y, p_{y} z, p_{z}\right) .
$$

Now the classical ground-state phase space distribution in Eq. (38) and the quantum ground-state wave function in Eq. (39) are both rotationally invariant and so correspond to the identity representation of the rotation group. Indeed, applying the Poisson bracket to find $L^{2}$ acting on the classical ground state phase space distribution $P_{0}\left(x, p_{x}, y, p_{y}, z, p_{z}\right)$, we have

$$
L^{2}=\left(y p_{z}-z p_{y}\right)^{2}+\left(z p_{x}-x p_{z}\right)^{2}+\left(x p_{y}-y p_{x}\right)^{2}
$$




$$
\begin{aligned}
\frac{\partial\left(L^{2}\right)}{\partial x} & =-2 p_{z}\left(z p_{x}-x p_{z}\right)+2 p_{y}\left(x p_{y}-y p_{x}\right)= \\
= & 2 x\left(p_{y}^{2}+p_{z}^{2}\right)-2 p_{x}\left(z p_{z}+y p_{y}\right) \\
\frac{\partial\left(L^{2}\right)}{\partial p_{x}} & =2 z\left(z p_{x}-x p_{z}\right)-2 y\left(x p_{y}-y p_{x}\right) \\
& =+2 p_{x}\left(y^{2}+z^{2}\right)-2 x\left(y p_{y}+z p_{z}\right),
\end{aligned}
$$

while from Eq. (38),

$$
\frac{\partial P_{0}}{\partial x}=m \omega_{0}^{2} x P_{0} \text { and } \frac{\partial P_{0}}{\partial p_{x}}=\frac{p_{x}}{m} P_{0}
$$

so that

$$
\left\{P_{0}, L^{2}\right\}=-\left(\frac{\partial L^{2}}{\partial x} \frac{\partial P_{0}}{\partial p_{x}}-\frac{\partial L^{2}}{\partial p_{x}} \frac{\partial P_{0}}{\partial x}+\frac{\partial L^{2}}{\partial y} \frac{\partial P_{0}}{\partial p_{y}}-\frac{\partial L^{2}}{\partial p_{y}} \frac{\partial P_{0}}{\partial y}+\frac{\partial L^{2}}{\partial z} \frac{\partial P_{0}}{\partial p_{z}}-\frac{\partial L^{2}}{\partial p_{z}} \frac{\partial P_{0}}{\partial z}\right)=0 .
$$

Also, if we have the quantum operator $\hat{L}^{2}$ act on the ground state wave function $\psi(x, y, z)$ in Eq. (39) using

$$
\hat{L}^{2} \psi_{0}(x, y, z)=-\hbar^{2}\left\{\left(y \frac{\partial}{\partial z}-z \frac{\partial}{\partial y}\right)^{2}+\left(z \frac{\partial}{\partial x}-x \frac{\partial}{\partial z}\right)^{2}+\left(x \frac{\partial}{\partial y}-y \frac{\partial}{\partial x}\right)^{2}\right\} \psi(x, y, z)
$$

we find

$$
\hat{L}^{2} \psi_{0}(x, y, z)=0
$$

Thus indeed, the ground states provides a basis for the identity representation of the threedimensional rotation algebra corresponding to $l=0$.

\section{Interpretation of the Physical Angular Momentum Squared in the Ground} State

The spherically-symmetric ground state of the three-dimensional harmonic oscillator is invariant under all the infinitesimal generators of rotation. It also corresponds to a Casimir operator value of $l=0$. However, just as the quantum theory and classical theory differ in their interpretation of the fluctuations in the energy of the ground state, the classical and quantum theories differ in their interpretations of the physical angular momentum. In the quantum theory, the hermitian operator $\hat{L}^{2}$ is connected to the physical angular momentum, 
and the average value for the square of the angular momentum of the system in the ground state follows from Eq. (47) as

$$
\left\langle\hat{L}^{2}\right\rangle_{0 \text { quantum }}=\int_{-\infty}^{\infty} d^{3} x \psi_{0}^{*} \hat{L}^{2} \psi_{0}=0
$$

On the other hand, in the classical theory, the numerical value for the angular momentum squared is not obtained from the algebra of the Poisson bracket acting on phase space as in Eq. (45), but rather is obtained by averaging over the ground state phase space distribution for the harmonic oscillator. We notice that

$$
\begin{aligned}
\left\langle L_{x}^{2}\right\rangle_{0 \text { classical }} & =\int_{-\infty}^{\infty} d^{3} p \int_{-\infty}^{\infty} d^{3} x\left(y p_{z}-z p_{y}\right)^{2} P_{0}\left(x, p_{x}, y, p_{y} z, p_{z}\right) \\
& =\int_{-\infty}^{\infty} d^{3} p \int_{-\infty}^{\infty} d^{3} x\left(y^{2} p_{z}^{2}+z^{2} p_{y}^{2}-2 y z p_{y} p_{z}\right) P_{0}\left(x, p_{x}, y, p_{y} z, p_{z}\right) \\
& =2\left(\frac{\hbar}{2 m \omega_{0}}\right)\left(\frac{\hbar m \omega_{0}}{2}\right)=\frac{\hbar^{2}}{2}
\end{aligned}
$$

with the same values for $\left\langle L_{y}^{2}\right\rangle_{0 \text { classical }}$ and $\left\langle L_{z}^{2}\right\rangle_{0 \text { classical }}$ Accordingly, we find

$$
\left\langle L^{2}\right\rangle_{0 \text { classical }}=\left\langle L_{x}^{2}+L_{y}^{2}+L_{z}^{2}\right\rangle_{0 \text { classical }}=\int_{-\infty}^{\infty} d^{3} p \int_{-\infty}^{\infty} d^{3} x L^{2} P_{0}\left(x, p_{x}, y, p_{y} z, p_{z}\right)=\frac{3}{2} \hbar^{2} .
$$

Thus the algebraic aspects associated with the representations of the rotation group appearing in Eqs. (45) and (47) agree between the classical and quantum theories. However, the average values for the square of the angular momentum of the physical system are given in Eqs. (48) and (50), and these do not agree between the classical and quantum theories. In the physical interpretation based upon the classical theory with zero-point radiation, the angular momentum of the particle in the ground states changes gradually due to interaction with the random zero-point radiation; sometimes the particle is moving around the potential center thereby giving a non-zero value for $\left\langle L^{2}\right\rangle_{0}$ classical. Thus in the classical interpretation where the phase space distribution arises from the random classical zero-point radiation, the mean square of the angular momentum does not vanish. On the other hand, the quantum result $\left\langle\hat{L}^{2}\right\rangle_{0 \text { quantum }}=0$ (involving the vanishing square of the angular momentum in the quantum ground state) is sometimes given a semiclassical interpretation with the suggestion that the particle is always moving directly toward the potential center or else directly away, but never going around the potential center. Whether this distinction is experimentally measurable at the microscopic level is not obvious. 


\section{CLASSICAL AND QUANTUM THEORIES AT POSITIVE TEMPERATURE}

\section{A. Continuity of the Classical Analysis}

It is interesting to see the classical-quantum connections extended to equilibrium situations involving positive temperature. The classical theory makes no change in the conceptual framework as the temperature is increased above absolute zero. Thus at positive temperature, the classical phase space distribution merely changes from Eq. (26) over to Eq. (25) because the thermal radiation has changed. However, the classical mechanical phase space distribution at temperature $T>0$ still corresponds to the equilibrium situation in the thermal radiation corresponding to Planck's spectrum including zero-point radiation. For the one-dimensional harmonic oscillator, Eqs. (5) and (25) give

$$
\langle H\rangle_{T \text { classical }}=\int d x d p_{x} H P_{T}\left(x, p_{x}\right)=\frac{1}{2} \hbar \omega_{0} \operatorname{coth}\left(\frac{\hbar \omega_{0}}{2 k_{B} T}\right) .
$$

The average value of the classical mechanical oscillator energy changes continuously from the value at zero temperature because the phase space distribution has changed continuously. The fluctuations in energy again follow from the classical phase space probability distribution. Thus we find

$$
\left\langle H^{2}\right\rangle_{T \text { classical }}=\int d x d p_{x} H^{2} P_{T}\left(x, p_{x}\right)=2\left(\frac{1}{2} \hbar \omega_{0} \operatorname{coth}\left(\frac{\hbar \omega_{0}}{2 k_{B} T}\right)\right)^{2}=2\langle H\rangle_{T \text { classical }}^{2} .
$$

\section{B. Change in the Quantum Analysis}

In contrast to the classical situation, the quantum situation undergoes a significant change. The quantum state can no longer be characterized by a single vector on the Hilbert space. Rather, the expectation value for a quantum operator at positive temperature is given as a sum over the expectation values in all the excited states with a weighting corresponding to the Boltzmann factor. For example, the expectation value of the energy is given by

$$
\langle\hat{H}\rangle_{T \text { quantum }}=\sum_{i}\left(\int_{-\infty}^{\infty} d^{3} x \psi_{i}^{*} \hat{H} \psi_{i}\right) \frac{\exp \left[-\mathcal{E}_{i} /\left(k_{B} T\right)\right]}{Z},
$$

where the probability normalization requires the normalization factor

$$
Z=\sum_{i} \exp \left[-\mathcal{E}_{i} /\left(k_{B} T\right)\right]
$$


For the case of the one-dimensional harmonic oscillator, the sum in Eq. (54) becomes a geometric series giving

$$
Z=\frac{1}{2 \sinh \left[\hbar \omega_{0} /\left(2 k_{B} T\right)\right]}
$$

and we find, by taking the derivative of the geometric series with respect to $\left(k_{B} T\right)^{-1}$,

$$
\begin{aligned}
\langle\hat{H}\rangle_{T \text { quantum }} & =\frac{1}{Z} \sum_{n=0}^{\infty}\left(n+\frac{1}{2}\right) \hbar \omega_{0} \exp \left[-(n+1 / 2) \hbar \omega_{0} /\left(k_{B} T\right)\right] \\
& =\frac{1}{2} \hbar \omega_{0} \operatorname{coth}\left(\frac{\hbar \omega_{0}}{2 k_{B} T}\right) .
\end{aligned}
$$

Thus again at positive temperature, the average value of the energy of the harmonic oscillator agrees between classical and quantum theories. On the other hand, the quantum energy fluctuations for the oscillator involve

$$
\begin{aligned}
\left\langle\hat{H}^{2}\right\rangle_{T \text { quantum }} & =\sum_{i}\left(\int_{-\infty}^{\infty} d^{3} x \psi_{i}^{*} \hat{H}^{2} \psi_{i}\right) \frac{\exp \left[-\mathcal{E}_{i} /\left(k_{B} T\right)\right]}{Z} \\
& =\frac{1}{Z} \sum_{n=0}^{\infty}\left[\left(n+\frac{1}{2}\right) \hbar \omega_{0}\right]^{2} \exp \left[-(n+1 / 2) \hbar \omega_{0} /\left(k_{B} T\right)\right] \\
& =2\left(\frac{1}{2} \hbar \omega_{0} \operatorname{coth}\left(\frac{\hbar \omega_{0}}{2 k_{B} T}\right)\right)^{2}-\left(\frac{1}{2} \hbar \omega_{0}\right)^{2} .
\end{aligned}
$$

Once again the fluctuations in the energy of the harmonic oscillator differ between classical and quantum theories. However, the discrepancy between $\left\langle H^{2}\right\rangle_{T \text { classical. }}$ and $\left\langle\hat{H}^{2}\right\rangle_{T \text { quantum }}$ in the classical and quantum theories at any temperature $T$ involves exactly the same discrepancy which existed at zero temperature,

$$
\left\langle H^{2}\right\rangle_{T \text { classical. }}-\left\langle\hat{H}^{2}\right\rangle_{T \text { quantum }}=\left(\hbar \omega_{0} / 2\right)^{2}
$$

The positive temperature aspect brings the classical and the quantum calculations closer together for the average value of the energy squared. On the other hand, as the temperature goes to zero, we find $\left\langle\hat{H}^{2}\right\rangle_{T \text { quantum }} \rightarrow 2\left(\hbar \omega_{0} / 2\right)^{2}-\left(\hbar \omega_{0} / 2\right)^{2}=\left(\hbar \omega_{0} / 2\right)^{2}=\langle\hat{H}\rangle_{0 \text { quantum }}^{2}$, which is the result in Eq. (35).

\section{PLANCK SPECTRUM OF QUANTUM THERMAL RADIATION}

The change in the quantum mechanical procedure for the harmonic oscillator in going from zero temperature to positive temperature is the same sort of shift which appears for quantum thermal radiation. According to the textbooks of modern physics and of quantum 
statistical mechanics, [23] the quantum vacuum at zero temperature involves virtual radiation fluctuations but no real photons. On the other hand, positive temperature indeed involves real photons with a radiation energy per normal mode $\mathcal{E}_{P}(\omega, T)$ given by Planck's spectrum without zero-point radiation, [23]

$$
\mathcal{E}_{P}(\omega, T)=\frac{\hbar \omega}{\exp \left[\hbar \omega /\left(k_{B} T\right)\right]-1}=\frac{1}{2} \hbar \omega_{0}\left[\operatorname{coth}\left(\frac{\hbar \omega_{0}}{2 k_{B} T}\right)-1\right] .
$$

Thus the quantum theory regards thermal radiation as vanishing at zero-temperature, $\mathcal{E}_{P}(\omega, T) \rightarrow 0$ as $T \rightarrow 0$. This is exactly the same view as was taken by the classical physicists working with Boltzmann statistical mechanics at the end of the 19th century. This viewpoint that the thermal spectrum vanishes at zero temperature is quite different from the classical view which includes classical zero-point radiation as an integral part of the spectrum of classical thermal radiation. [24] In classical theory, the random classical zero-point radiation leads to the zero-point fluctuations of a classical mechanical oscillator which must match the average radiation energy at the natural frequency of the oscillator in order to be in equilibrium with the random classical radiation. On the other hand, quantum theory has an entirely different basis for thermal equilibrium between radiation and matter. The quantum harmonic oscillator has a mechanical zero-point energy $\hbar \omega_{0} / 2$ in Eq. (33) which does not appear in the quantum Planck radiation spectrum Eq. (59) which has no zero-point energy in quantum theory.

\section{SQUARE OF THE ANGULAR MOMENTUM AT POSITIVE TEMPERA- TURE}

It is also interesting to see the extension of these ideas to the situation involving the isotropic harmonic oscillator at strictly positive temperature T. 25] The classical theory treats all random radiation (both zero-point radiation and thermal radiation above the zero-point radiation) as thermal radiation contributing to the random particle motion on phase space, as indicated in Eq. (25). At positive temperature, the classical phase space remains spherically symmetric and corresponds to the identity representation of the rotation group; accordingly, the classical Poisson bracket of $L^{2}$ applied to the phase space remains $\left\{P_{T}, L^{2}\right\}=0$, as in Eq. (45). The average value of the angular momentum squared is still obtained by averaging over the phase space as in Eq. (50) (but now with the phase space 
associated with positive temperature as in Eq. (25) ), and becomes

$$
\left\langle L^{2}\right\rangle_{T \text { classical }}=(3 / 2) \hbar^{2} \operatorname{coth}^{2}\left[\hbar \omega_{0} /\left(2 k_{B} T\right)\right]
$$

On the other hand, for positive temperature $T>0$, the quantum theory no longer represents the physical system in thermal equilibrium by a single vector in Hilbert space, but rather treats the behavior associated with strictly positive temperature in a fashion quite different from the expectation value calculation in Eq. (48). Now a sum over the excited eigenstates of energy $\mathcal{E}_{i}$ is required with a weighting by the Boltzmann factor,

$$
\left\langle\hat{L}^{2}\right\rangle_{T \text { quantum }}=\sum_{i}\left(\int_{-\infty}^{\infty} d^{3} x \psi_{i}^{*} \hat{L}^{2} \psi_{i}\right) \frac{\exp \left[-\mathcal{E}_{i} /\left(k_{B} T\right)\right]}{Z} .
$$

The average angular momentum operator squared involves the evaluation of terms of the form[25] $\left\langle\hat{x}^{2}\right\rangle_{T}=\left[\hbar /\left(2 m \omega_{0}\right] \operatorname{coth}\left[\hbar \omega_{0} /\left(2 k_{B} T\right], \quad\left\langle\hat{p}^{2}\right\rangle_{T}=\right.\right.$ $\left[\hbar m \omega_{0} / 2\right] \operatorname{coth}\left[\hbar \omega_{0} /\left(2 k_{B} T\right], \quad\langle\hat{x} \hat{p}\rangle_{T}=i \hbar / 2\right.$, and $\langle\hat{p} \hat{x}\rangle_{T}=-i \hbar / 2$. The result for the expectation value for the square of the angular momentum is

$$
\left\langle\hat{L}^{2}\right\rangle_{T \text { quantum }}=(3 / 2) \hbar^{2} \operatorname{coth}^{2}\left[\hbar \omega_{0} /\left(2 k_{B} T\right)\right]-(3 / 2) \hbar^{2}
$$

The quantum excited states no longer belong exclusively to the identity representation of the rotation group with $l=0$, but now include states with representations with $l>0$, and so now give non-zero values for the angular momentum squared. We notice that the difference between the classical and the quantum average values of the angular momentum squared always differ by the same amount $(3 / 2) \hbar^{2}$ which appeared at zero temperature

$$
\left\langle L^{2}\right\rangle_{T \text { classical }}-\left\langle\hat{L}^{2}\right\rangle_{T \text { quantum }}=(3 / 2) \hbar^{2} .
$$

At high temperature $\hbar \omega_{0}<<k_{B} T$, we find the limit $\left(\hbar \omega_{0} / 2\right) \operatorname{coth}\left[\hbar \omega_{0} /\left(2 k_{B} T\right] \rightarrow k_{B} T\right.$, so that the classical phase space distribution in Eq. (25) becomes the Boltzmann probability distribution of traditional classical statistical mechanics. The classical result for the average of the square of the angular momentum in Eq. (마) goes over to $\left\langle L^{2}\right\rangle_{T \text { classical }} \approx 6\left(k_{B} T / \omega_{0}\right)^{2}$ which is the value obtained from traditional classical statistical mechanics where Planck's constant has completely disappeared from the expression. The quantum result becomes $\left\langle\hat{L}^{2}\right\rangle_{T \text { quantum }} \approx 6\left(k_{B} T / \omega_{0}\right)^{2}-(3 / 2) \hbar^{2}$ which still subtracts the value $(3 / 2) \hbar^{2}$ associated with zero temperature. 


\section{CLOSING SUMMARY}

In this article, we have explored some connections between classical and quantum theories for the harmonic oscillator. As pointed out by Dirac, the classical-quantum connection between Poisson brackets and quantum commutators means that the theories agree on the basic algebraic structure associated with groups of transformations. Dirac's connection to the quantum operators on Hilbert space also gives well-defined numerical values tied to Planck's constant $\hbar$ for the expectation values of the quantum operators. On the other hand, the Poisson brackets provide canonical transformations, but no numerical values for the physical quantities in the classical theory. The classical theory must go outside nonrelativistic classical mechanics in order to introduce a fundamental scale for the classical physical quantities. One natural extension looks to classical electrodynamics, where Planck's constant $\hbar$ can assume a natural role as the scale factor for the Lorentz-invariant classical zero-point radiation which forms a natural part of classical thermal radiation. The associated electromagnetic theory is often termed "stochastic electrodynamics," and can provide classical explanations for Casimir forces, van der Waals forces, oscillator specific heats, diamagnetism, blackbody radiation, and the absence of atomic collapse.[14] Any mechanical system located in random classical radiation will inherit the radiation scale involving $\hbar$, and will come to equilibrium with a phase space distribution corresponding to a classical stochastic mechanical theory. We have used the classical stochastic mechanical theory for our classical analysis in this article. For the harmonic oscillator, the classical-quantum connections involve agreement regarding average values, but disagreement regarding fluctuations. In all cases, the classical behavior fits with fundamental classical ideas, which can be quite different from quantum ideas. In some cases, the classical results are reassuring. For example, the positive value for the square of the angular momentum of the three-dimensional oscillator is more comforting to classical sensibilities than is the zero-value claimed by the orthodox interpreters of the quantum theory.

[1] P. A. M. Dirac, "The Fundamental Equations of Quantum Mechanics," Proc. Roy. Soc. A 109, 642-653 (1925).

[2] P. A. M. Dirac, The Principles of Quantum Mechanics 4th ed. (Oxford at the Clarendon Press 
1958), Chapter IV, pp. 87; L. I. Schiff, Quantum Mechanics 3rd ed. (McGraw-Hill, New York 1968), pp. 175-177; L. E. Ballentine, Quantum Mechanics (Prentice Hall, Englewood Cliffs, NJ 1990), pp. 67-68; J. J. Sakurai, Modern Quantum Mechanics Revised Edition (Addison-Wesley, New York 1994), pp. 50-51.

[3] J. P. Dahl, "Physical origin of the Runge-Lenz vector," J. Phys. A: Gen. 30, 6831-6840 (1997), notes that the "relation $\ldots$ is generally valid when $[f, g]$ may be written as a linear combination of Poisson brackets between functions of which at least one is no more than quadratic in the components of $\mathbf{r}$ or $\mathbf{p . "}$

[4] T. H. Boyer, "The contrasting roles of Planck's constant in classical and quantum theories," Am. J. Phys. 86, 280-283 (2018).

[5] T. H. Boyer, "Blackbody radiation in classical physics: A historical perspective," Am. J. Phys. 86, 495-509 (2018).

[6] See for example, D. J. Griffiths, Introduction to Quantum Mechanics 2nd ed. (Pearson Prentice Hall, Upper Saddle River, NJ 2005), p. 43.

[7] See, for example, H. Goldstein, Classical Mechanics 2nd edn, (Addison-Wesley, Reading, MA 1981), beginning on p. 397.

[8] See, for example, Sakurai in ref. 2, pp. 50-51.

[9] See, for example, A. Zee, Group Theory in a Nutshell for Physicists (Princeton U. P., Princeton 2016), p. 210.

[10] See, for example, Griffiths in ref. 6, p. 42.

[11] See, for example, Griffiths in ref. 6, p. 46.

[12] See for example, H. Goldstein in ref. 7, p. 421.

[13] W. Pauli, "Über das Wasserstoffspecktrum vom Standpunkt der neuen Quantenmechanik," Zeits. für Phys. 36, 336-363 (1926).

[14] The classical point of view begins with the careful work of T. W. Marshall, "Random electrodynamics," Proc. R. Soc. A276, 475-491 (1963); "Statistical Electrodynamics," Proc. Camb. Phil. Soc. 61, 537-546 (1965). For recent work, see T. H. Boyer, "Stochastic Electrodynamics: The Closest Classical Approximation to Quantum Theory," Atoms 7(1), 29-39 (2019); W. C-W. Huang and H. Batelaan, "Discrete Excitation Spectrum of a Classical Harmonic Oscillator in Zero-Point Radiation," Found. Phys. 45, 333-353 (2015); D. C. Cole and Y. Zou, "Quantum Mechanical Ground State of Hydrogen Obtained from Classical Electrodynamics," 
Phys. Lett. A 317, 14-20 (2003). A review of the work on classical electromagnetic zero-point radiation up to 1996 is provided by L. de la Pena and A. M. Cetto, The Quantum Dice An Introduction to Stochastic Electrodynamics (Kluwer Academic, Dordrecht 1996). Further suggestions linking quantum mechanics and zero-point radiation appear in the work of L. de la Pena, A. M. Cetto, and A. V. Hernandez, The Emerging Quantum: The Physics Behind Quantum Mechanics (Springer, New York 2015).

[15] See for example, M. Planck, The Theory of Heat Radiation (Dover, New York 1959).

[16] H. B. G. Casimir, "On the attraction between two perfectly conducting plates," Proc. Ned. Akad. Wetenschap. 51, 793-795 (1948).

[17] M. J. Sparnaay, "Measurement of the attractive forces between flat plates," Physica (Amsterdam) 24, 751-764 (1958); S. K. Lamoreaux, "Demonstration of the Casimir force in the 0.6 to $6 \mu \mathrm{m}$ range," Phys. Rev. Lett. 78, 5-8 (1997): 81, 5475-5476 (1998); U. Mohideen, "Precision measurement of the Casimir force from 0.1 to $0.9 \mu \mathrm{m}$," ibid. 81, 4549-4552 (1998); H. B. Chan, V. A. Aksyuk, R. N. Kleinman, D. J. Bishop, and F. Capasso, "Quantum mechanical actuation of microelectromechanical systems by the Casimir force," Science 291, 1941-1944 (2001): G. Bressi, G. Carugno, R. Onofrio, and G. Ruoso, "Measurement of the Casimir force between parallel metallic surfaces," Phys. Rev. Lett. 88, 041804(4) (2002).

[18] T. H. Boyer, "Retarded van der Waals Forces at All Distances Derived from Classical Electrodynamics with Classical Electromagnetic Zero-Point Radiation," Phys. Rev. A7, 1832-1840 (1973).

[19] See, for example, T. H. Boyer, "Random electrodynamics: The theory of classical electrodynamics with classical electromagnetic zero-point radiation," Phys. Rev. D 11, 790-808 (1975).

[20] See, for example, the work of T. W. Marshall (1963) in ref. 14, or T. H. Boyer in ref. 19, or B. H. Lavenda, Statistical Physics: A Probabilistic Approach (Wiley, New York 1991), pp. 73-74.

[21] T. H. Boyer, "General connection between random electrodynamics and quantum electrodynamics for free electromagnetic fields and for dipole oscillator systems," Phys. Rev. D 11, 809-830 (1975).

[22] For a graph showing the gradual change in the classical oscillator energy when interacting with zero-point radiation in a simulation, see W. Huang and H. Batelaan, "Dynamics Underlying the Gaussian Distribution of the Classical Harmonic Oscillator in Zero-Point Radiation," J. 
Comp. Methods Phys. 2013, 308538 (2013).

[23] See for example, R. Eisberg and R. Resnick, Quantum Physics of Atoms, Molecules, Solids, Nuclei, and Particles 2nd edn (Wiley, New York 1985); K. S. Krane, Modern Physics 2nd edn (Wiley, New York 1996); R. Taylor, C. D. Zafiratos, and M. A. Dubson, Modern Physics for Scientists and Engineers 2nd edn (Pearson, New York, 2003); S. T. Thornton and A. Rex, Modern Physics for Scientists and Engineers 4th edn (Brooks/Cole, Cengage Learning, Boston, MA, 2013).

[24] T. H. Boyer, "Understanding zero-point energy in the context of classical electromagnetism," Eur. J. Phys. 37, 055206(14) (2016); "Thermodynamics of the harmonic oscillator: derivation of the Planck blackbody spectrum from pure thermodynamics," Eur. J. Phys. 40, 025101(16pp) (2019); "Diamagnetic behavior in random classical radiation," Am. J. Phys. 87, 915-923 (2019); "Particle Brownian motion due to random classical radiation: Superfluid-like behavior in classical zero-point radiation," Eur. J. Phys. (to be published), https://doi.org/10.1088/1361-6404/ab988d.

[25] We can calculate the results for $\left\langle L^{2}\right\rangle_{T}$ by using the average values for powers of displacements and momenta at positive temperature quoted in Table II of ref. 21. 